\section{A APLICAÇÃO DO STIPER EM PONTOS DA REFLEXOLOGIA PODAL EM CASOS DE DOR LOMBAR}

\section{STIPER APPLICATION ON FEET REFLEXOLOGY POINTS IN CASES OF LOMBAR PAIN}

\section{RESUMO}

Este estudo tem como objetivo verificar a eficácia da aplicação do Stiper sobre pontos da reflexoterapia em casos de dor lombar. O Stiper é uma pastilha de óxido de silício que tem a função de estimular pontos, zonas ou áreas do corpo a partir da reordenação da frequência energética local. A pesquisa justifica-se por viabilizar a utilidade deste método terapêutico como extensão do tratamento naturológico fora de consultório, promovendo estímulo terapêutico realizado pelo profissional por mais tempo. Participaram efetivamente, até o final da pesquisa, 43 (quarenta e três) pessoas que relatavam ter dores na região lombar para receber aplicações do Stiper. Foram realizados seis encontros, na frequência de uma vez por semana. Para análise dos resultados foram utilizados: escala facial de dor Wong-Baker (para identificar intensidade de dor) em todas as sessões e Questionário Oswestry (para avaliar a incapacidade causada pela dor lombar) aplicado no primeiro e último dias da pesquisa. Os resultados foram satisfatórios tanto na redução do nível de dor quanto na de incapacidade gerada pela mesma. Conclui-se que a associação da reflexoterapia e do Stiper, através dos questionários utilizados, possui resultados estatisticamente e clinicamente significativos quanto à analgesia da região lombar uma vez que durante a pesquisa obteve-se como resultado $72 \%$ de redução no nível de dor e 55\% de melhora na incapacidade gerada pela dor.

\section{PALAVRAS-CHAVE}

Reflexologia.

Dor Lombar.

Stiper.

\section{Cristina de Paula Avelino \\ - Possui graduação em Naturologia Aplicada pela Universidade do Sul de Santa Catarina (2012). Tem experiência na área de Saúde Coletiva, com ênfase em Acupuntura.}

Graciela Mendonça da Silva de Medeiros - Enfermeira obstetra graduada pela Universidade do Sul de Santa Catarina- UNISUL (1993-1997). Especialista em Acupuntura e técnicas da Medicina Tradicional Chinesa pela Faculdade de Tecnologia em saúde CIEPH-Atualmente é docente do curso de Naturologia Aplicada da Universidade do Sul de Santa Catarina-UNISUL; Centro Técnico Harmonie e do Centro integrado de Estudos e Pesquisas do Homem.

CORRESPONDENTE

Graciela Mendonça da Silva de Medeiros Av. Pedra Branca $n^{\circ}$ 25, Palhoça - SC

Recebido: $12 / 09 / 12$

Aprovado: 22/11/12 


\begin{abstract}
This study aims to determine the effects of Stiper application on reflexology points in cases of low back pain. The Stiper is a wafer of silicon oxide which works stimulating points, zones, or body areas from reordering the local frequency energy. This research is justified by enabling the use of this therapeutic method as an extension of Naturólogo's work out of office, promoting a longer stimulation effect therapy. 43 (forty three) people joined effectively this study. All of them complained about low back pain and received the Stiper application on their feet. There were 6 (six) meetings, one per week, for 6 (six) weeks. For data analysis were used: Wong Baker Faces Pain Scale (to quantify pain intensity) in every session and the Oswestry Questionnaire (to identify the disability caused by back pain) applied on the first and last days of the study. The results were satisfactory both in reducing the level of pain and disability. The conclusion is that the combination of reflexotherapy and Stiper application through the chosen questionnaires in this research, shows statistically and clinically significant analgesia in the lumbar region.
\end{abstract}

KEYWORDS: Reflexology, Back pain, Stiper.

\section{INTRODUÇÃO}

A Naturologia Aplicada é uma área das ciências biológicas e da saúde que se propõe a atuar de forma integrativa na manutenção, recuperação, promoção da saúde e da qualidade de vida de seus interagentes ${ }^{16,17}$. Desta forma, o naturólogo não se presta apenas ao tratamento de patologias, como também à promoção da saúde através das práticas naturais, associadas a um processo de interação entre o naturólogo e o interagente, a fim de influir positivamente na qualidade de vida individual ${ }^{9}$.

O naturólogo, na interagência, engloba os níveis físicos, energéticos, emocionais e mentais dos indivíduos, no qual há a participação ativa, tanto do naturólogo quanto do interagente, em um processo reflexivo e avaliativo. Este profissional utiliza como forma de tratamento as Práticas Integrativas Complementares (PIC), e sustenta seu tratamento nas medicinas tradicionais chinesa, ayurvédica e xamânica, as quais entendem o indivíduo como um ser único e que permanece em constante interação com o meio no qual está inserido.

Uma das práticas naturais empregadas pelo naturólogo é a reflexoterapia. O termo reflexologia vem do grego logos e é a área do conhecimento que estuda os pontos reflexos dos pés, mãos e orelhas e os efeitos de seus estímulos no organismo humano. Através dela é possível identificar possíveis distúrbios físicos e emocionais relacionados a sinais vi- suais e de sensibilidade à dor destes locais ${ }^{25}$. Sendo assim, a reflexoterapia é a aplicação prática dos conhecimentos da reflexologia.

Além de identificar a existência de distúrbios, a reflexoterapia também se propõe a tratá-los através de manipulações sobre as áreas reflexas específicas, permitindo ao cérebro detectar, com maior eficiência possível, problemas no organismo e assim iniciar o processo de cura ${ }^{25}$.

Um dos métodos recentes de estímulo dos pontos reflexos é o uso do Stiper. Trata-se de uma manta hipoalergênica preenchida por silicatos (óxido de silício) na forma de pastilhas que são utilizadas com a finalidade de equilibrar a frequência energética dos pontos onde são aderidas causando um aumento de energia em casos de deficiência e reduzindo o excesso da mesma no caso inverso $0^{3}$. O Stiper pode ser usado tanto em pontos de acupuntura quanto nos pontos reflexos, obtendo os mesmos efeitos terapêuticos que o estímulo com a agulha ou pressão manual podem gerar ${ }^{21}$.

O Stiper é um produto que possibilita a extensão das terapias realizadas pelo naturólogo, já que, por estar fixado ao corpo, é uma forma de continuar o tratamento fora do consultório, mantendo um estímulo durante horas e até dias, podendo ser utilizado para muitos tipos de distúrbios, inclusive dores.

A dor é considerada, além de um mecanismo de defesa, um reflexo protetor do organismo para evitar 
danos teciduais ou possíveis ameaças ${ }^{20}$. Através dela toma-se conhecimento dos desequilíbrios e doenças do corpo. Ela desencadeia um conjunto de reações de adaptação, de ordem psicológica, autonômica e motora, visando afastar o organismo da causa da agressão e, assim, preservá-lo ${ }^{24,31,35}$.

A dor lombar não é uma doença por si só, mas um sintoma ${ }^{19}$. As dores lombares estão entre as mais comuns nas cidades industrializadas e acometem mais de $80 \%$ da população em ambos os $\operatorname{sexos}^{28}$. Ela aumenta à medida que a idade aumenta, apresentando maior prevalência em um grupo etário acima dos 40 anos $^{30}$. Desta forma, tornou-se a causa mais comum de incapacidade crônica e uma das razões mais comuns para a aposentadoria prematura. No Reino Unido, com uma população de cerca de 58 milhões de habitantes, houve perda de dias de trabalho estimada em 52 milhões e pagamento de 105 milhões de benefícios pela previdência social, relacionados à dor nas costas, em 1993. Números semelhantes, proporcionais à população, são encontrados por toda a sociedade ocidental ${ }^{6}$.

A lombalgia pode ser descrita como dor na coluna lombar, dor no quadril ou uma combinação des$\operatorname{tas}^{28}$. Ela pode se fazer presente de forma localizada ou irradiada, sendo originada de muitas estruturas. As principais causas são as lesões musculoesqueléticas e os processos degenerativos de disco e facetas ${ }^{1}$. Frequentemente a dor é acompanhada de contratura muscular e dificuldade nos movimentos segmentares da região atingida. Na maioria dos casos, a dor é um reflexo da ruptura de um disco intervertebral. Esta provoca um processo inflamatório gerador de irritação do nervo de alarme regional que causa ainda mais dor e contratura dos músculos lombares ${ }^{31}$.

Em relação à duração, as dores lombares podem ser classificadas como agudas ou crônicas ${ }^{4}$. Em termos médicos, a dor aguda tem início rápido, limitando o indivíduo a uma atividade ou situação. Ela surge a partir de um movimento aparentemente normal e que não geraria dor, mas esta começa provocando deficiência no movimento, o qual poderá ser o primeiro episódio doloroso ${ }^{4}$. A dor aguda é uma resposta normal causada por uma lesão de tecido com con- sequente ativação dos nociceptores no local da lesão. Ela desaparece antes mesmo do restabelecimento do tecido lesado. Este tipo de dor geralmente aparece após cirurgias ou situações traumáticas e persiste menos de um mês, possuindo também caráter protetor. Nestes casos, normalmente o tratamento é feito pela administração de analgésicos, anti-inflamatórios e repouso ${ }^{13,32,34}$.

A dor crônica ocorre quando os mecanismos de defesa para controle da dor não funcionam adequadamente, ou quando determinadas doenças associadas à dor tornam-se crônicas por razões desconhecidas. Ela pode durar meses ou anos, causando um significativo impacto sobre a qualidade de vida do paciente, interferindo na homeostase do mesmo e na

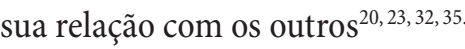

Vários pesquisadores caracterizam a dor lombar crônica como uma doença de pessoas com vida sedentária. A inatividade física estaria relacionada direta ou indiretamente a dores na coluna. Pode-se considerá-las um subproduto da combinação da aptidão músculo-esquelética deficiente e uma ocupação que força essa região ${ }^{7,34}$. Contudo, outros fatores como tabagismo, insatisfação laboral, grau de escolaridade, síndromes depressivas, litígio trabalhistas, fatores genéticos e antropológicos, alterações climáticas, modificações de pressão atmosférica e temperatura também podem contribuir para o surgimento desta patologia ${ }^{5}$ sendo que em $80 \%$ dos casos o diagnóstico etiológico é praticamente impossível de se determinar ${ }^{13}$.

Visto que inúmeras condições podem gerar esta disfunção, os tratamentos sugeridos para tal possuem três objetivos: alívio da dor, aumento da capacidade funcional e o retardamento da progressão da doenç $\mathrm{a}^{27}$. Desta forma, já existem vários fatores que vêm se transformando e alterando o risco desta patologia, dentre eles a ergonomia nas empresas, que, adequando o ambiente de trabalho ao trabalhador, facilita a execução de tarefas, faz com que os mesmos utilizem menos força, gerando menor sobrecarga estática ${ }^{13}$. Exercícios aeróbicos e de fortalecimento da musculatura paravertebral também são comprovadamente eficazes ${ }^{5}$. 
Muitas terapias não farmacológicas estão disponíveis para o tratamento de lombalgias crônicas ${ }^{8}: 0$ tratamento conservador de repouso, como o da lombalgia aguda, porém por período maior do que três dias; fisioterapia; calor; gelo; massagem; ultrassom; eletroestimulação; coletes; e tratamentos alternativos como a hidroterapia, acupuntura, massoterapia e reflexoterapia ${ }^{13}$.

Com esta pesquisa, objetivou-se verificar o efeito da aplicação dos stipers em pontos da reflexologia podal em casos de dor lombar.

\section{MÉTODOS}

Esta pesquisa classificou-se de natureza preponderantemente quantitativa, do tipo experimental e explicativa, e mensura variáveis capazes de identificar os fatores que contribuíram para a ocorrência de dor lombar e investigou-se hipóteses para o esclarecimento da relação de causa-efeito quando tratados com o Stiper em pontos reflexológicos ${ }^{11,14,22}$.

Esta pesquisa baseou-se na resolução 196/96 do Conselho Nacional de Saúde por possuir como unidade experimental seres humanos. $\mathrm{O}$ projeto foi aprovado pelo Comitê de Ética e Pesquisa (CEP) da UNISUL, sob identificação do registro/ código:11.489.4.06.III, em 01 de dezembro de 2011.

Para a participação na pesquisa, foi solicitado preenchimento do TCLE que garante sigilo e anonimato aos participantes, assim como o direito de desistência da pesquisa a qualquer momento.

O estudo se realizou no CPN (Centro de Práticas Naturais), da UNISUL (Universidade do Sul de Santa Catarina), município de Palhoça, Santa Catarina, CEP 88137-270, de março a maio de 2012.

Os 50 (cinquenta) sujeitos foram selecionados por ordem de chegada, considerando os critérios de inclusão e exclusão da pesquisa. Os critérios de inclusão foram: possuir idade entre 18 a 65 anos e ter dor lombar constante há pelo menos 2 dias. Os critérios de exclusão foram: apresentar patologias de caráter maligno como câncer e lesões nos pés, tais como fissuras, fístulas, dermatites, dentre outras que danifiquem a integridade da pele. Também foi apresentado como critério de exclusão atingir um núme- ro abaixo de 10 no Questionário Oswestry de incapacidade. Este questionário é dividido em 10 partes, sendo elas com relação a intensidade da dor sentida, a capacidade de realização dos cuidados pessoais do entrevistado, a capacidade de carregar pesos, de andar, de sentar, de ficar de pé, de dormir, de ter uma vida social, de viajar e de realizar trabalhos de casa e emprego ${ }^{12}$.

Além deste, a escala facial de Wong Baker serviu como método para avaliar a dor dos participantes no momento dos encontros. Esta escala faz uso de imagens para facilitar a percepção do nível da dor e, assim, o paciente deve escolher a face correspondente ao seu estado atual.

Para as aplicações utilizou-se pastilhas de óxido de silício, chamadas de Stiper, fabricadas pela empresa STIPER (TBG EPP), micropore para fixá-las aos pontos desejados, algodão e álcool 70\%, para assepsia.

Iniciou-se a pesquisa com o preenchimento do Questionário Oswestry de incapacidade por dor lombar em uma das salas do CPN de acordo com o horário de cada sujeito. Tendo o resultado deste questionário igual ou maior que 10 (dez) pontos - uma vez que quanto maior a pontuação, maior o nível de dor -, foi realizada a marcação individual na Escala facial de dor Wong-Baker, que foi repetida em todos os encontros. Foi questionado também quanto à administração de analgésicos para o controle da dor, por quanto tempo a medicação foi utilizada, para então serem aplicadas as pastilhas Stiper.

Aplicaram-se 3 pastilhas Stiper na região correspondente à coluna lombar em cada pé e um Stiper nos pontos de tensão, um em cada pé, totalizando 8 Stipers por sessão (APÊNDICE A). Caso as pastilhas caíssem antes do período de cinco dias, foi pedido para que os participantes não tentassem recolocá-las para que não houvesse a possibilidade de os Stipers serem postos em lugares diferentes dos propostos pela pesquisa. Questionou-se sobre quanto tempo as pastilhas permaneceram nos pés antes de caírem, para melhor acompanhamento dos efeitos.

Foram 6 (seis) encontros ao total, uma vez por semana durante seis semanas. Destes apenas o últi- 
mo não teve a aplicação dos Stipers, mas foram realizadas as aplicações do questionário Oswestry de incapacidade por dor lombar e da escala facial.

O método utilizado para análise quantitativa dos dados obtidos foi o Teste T-Students pareado para comparação das médias e o teste do Qui-quadrado de tendência linear para as variáveis ordinais, a partir do programa Stata 11.0, considerando estatisticamente significativo quando $\mathrm{p} \leq 0,05$.

\section{RESULTADOS E DISCUSSÃO}

Para este estudo, apresentaram-se 50 inscritos, porém 7 optaram por desistir da pesquisa. Desta forma, restaram 43 participantes que mantiveram a frequência dos 6 encontros em 6 semanas para a realização da coleta dos dados, sendo 14 (32,56\%) do sexo masculino e $29(67,44 \%)$ do sexo feminino. A idade dos participantes variou de 21 a 61 anos, e a idade média foi de 38,6 anos. Dos 43 participantes, $33(76,74 \%)$ não relataram diagnóstico de patologias que pudessem ocasionar a dor lombar, enquanto $10(23,26 \%)$ participantes possuíam alguma doença ou lesão na região lombar previamente diagnosticada, sendo que a encontrada com maior frequência foi a hérnia de disco em 5 $(11,63 \%)$ pessoas (Tabela 1$)$. Como visto com tais dados, não é necessário que haja uma lesão física na coluna para que a dor apareça. A causa da maioria das dores lombares vem de alterações biomecânicas da coluna vertebral, provocadas por más posturas no trabalho e fora dele, fraqueza muscular, em especial dos músculos abdominais, sobrecarga mecânica, inflamação, obesidade, falta de exercício e maus hábitos posturais. Quando há estresse biomecânico por sobrecargas, tarefas altamente demandantes ou repetitivas, se apresenta a tensão muscular que produz fadiga e desencadeia processos neuroquímicos que podem gerar dor ${ }^{7}$.

A análise das escalas faciais Wong Baker mostrou que através da aplicação durante a pesquisa, podem-se notar resultados estatisticamente significativos quanto à diminuição das dores lombares momentâneas destes participantes, comparando cada um dos encontros com o primeiro (Tabela 2).
Também foi feita a análise dos dados do questionário Oswestry de Incapacidade por dor lombar e cada uma das seções deste será analisada separadamente.

Quanto à intensidade da dor, verificou-se que, no começo da pesquisa, 16 pessoas $(37,21 \%)$ faziam uso de analgésicos devido a intensidade da dor e este número foi reduzido para $3(6,98 \%)$ ao final da pesquisa (Tabela 3). Em alguns casos, os participantes relataram terem dores muito fortes, mas não poderem tomar analgésico por motivos diferentes. Isto pode ter ocasionado uma diferença com relação aos resultados pela ausência de uma resposta que equivalesse a casos isolados. Ademais, no começo da pesquisa apenas 1 pessoa $(2,33 \%)$ relatou ter uma dor não tão intensa enquanto que ao final da pesquisa, 24 dos participantes $(55,81 \%)$ assinalaram o mesmo.

Com relação aos cuidados pessoais, 21 participantes $(48,84 \%)$ sentiam dor em atividades tais como lavar os pés, amarrar o sutiã, amarrar os sapatos e escovar os dentes. Até o último encontro, o número de pessoas com tais dificuldades foi reduzido a $10(23,26 \%)$. Sendo assim, no primeiro encontro 22 pessoas $(51,16 \%)$ não sentiam dor ao se cuidar e no final da pesquisa este número subiu para $33(76,74 \%)$.

As modificações e dor na coluna lombar podem estar relacionadas a doenças ou a esforços excessivos sobre a coluna, como carregar peso, ou decorrentes de um movimento brusco de torção da coluna vertebral ${ }^{2}$. Sendo assim, quanto a capacidade de carregar pesos houve um impasse para muitos dos participantes, pois estes não carregavam pesos para saber de fato qual opção responder dentro do questionário. Contudo, verificou-se que, no começo da pesquisa, apenas 2 pessoas $(4,65 \%)$ eram capazes de carregar qualquer tipo de peso sem sentir dor enquanto que no último encontro $19(44,19 \%)$ relataram ser capazes de fazer o mesmo sem sentir dor.

No quesito andar, houve melhora em todas as opções do questionário, mostrando um aumento no percentual de pessoas que se tornaram capazes de caminhar qualquer distância sem provocar dor de $21(48,84 \%)$ para $34(79,07 \%)$. 
Os músculos da região lombar desempenham importante papel protetor das estruturas passivas da coluna vertebral. A hipotonicidade proveniente do desuso, a permanência prolongada em determinadas posições, como a posição sentada, causam uma transferência excessiva de carga a essas estruturas, provocando dor ${ }^{10}$. Desta forma, sentar provou-se um dos movimentos que mais gerava incômodo, dor e queimação na região lombar, exigindo que muitos dos participantes ficassem constantemente se movendo em seus assentos para encontrar uma posição na qual pudessem permanecer por um longo período uma vez que a maior parte dos sujeitos da pesquisa trabalhava/estudava nesta posição. Sendo assim, houve uma melhora significativa na diminuição da dor geral, visto que o número de pessoas que não referiram mais dor ao sentar-se em qualquer assento aumentou de 2 (4,65\%) para 17 (39,53\%) enquanto as que não referiram dor ao sentar-se em uma cadeira específica também aumentou de 3 (6,98\%) para $10(23,26 \%)$.

A posição ereta, exclusiva do ser humano, faz com que o homem possua uma maior predisposição a patologias da coluna, sendo a dor na região o sintoma experimentado em quase a sua totalidade quando uma lesão aparece $e^{23}$. Desta maneira, no quadro "ficar de pé" foi encontrado o mesmo problema que com os pesos, em que grande parte das pessoas não permanecia nesta posição por tempo o suficiente para saber quando a dor aparecia. No entanto, houve melhora de 1 (2,33\%) para 17 pessoas $(39,53 \%)$ do início para o final da pesquisa no número de sujeitos capazes de permanecer de pé sem sentir dor pelo tempo que quisesse.

Quanto às perguntas sobre o sono, grande parte das pessoas precisou adaptar suas respostas uma vez que a opção desejada não constava no questionário. No entanto, muitos dos participantes responderam "dormir bem com o uso de analgésicos" para dizer que na hora de se deitarem para dormir, sentiam dor, mas esta não impedia um sono de qualidade. Com isto, ao longo da pesquisa, não somente ao refazer o questionário Oswestry, alguns participantes relataram a melhora no sono, sendo capazes de dormir por mais horas, mas frequentemente acorda- vam com dores por terem permanecido demasiado tempo na posição deitada. Em números, no começo da pesquisa 15 pessoas $(34,88 \%)$ se deitavam para dormir sem sentir dor, enquanto que ao final 34 $(79,07 \%)$ relataram o mesmo.

Houve uma diferença positiva quanto aos níveis de dor relacionados a vida social dos participantes, demonstrando não somente que se tornaram capazes de realizar atividades sociais sem dor, como realizar atividades mais enérgicas como a dança e esportes sem prejuízos físicos. Em dados numéricos, o número de pessoas que não possuíam dor na vida social subiu de $17(39,53 \%)$ para $32(74,42 \%)$.

A seção que discute a dor durante viagens é pertinente, uma vez que grande parte das pessoas que participaram da pesquisa utilizavam meios de transporte como ônibus e carros para chegarem ao local de trabalho e estudo. No entanto, nem sempre era possível ficar sentado nos veículos ou o tempo de viagem se encaixava com as respostas propostas no questionário. Foi necessário adaptar a resposta dos participantes para realizar esta parte da pesquisa. No entanto, houve melhora de $6(13,95 \%)$ para 15 $(34,88 \%)$ na ausência de dor nesta atividade.

A dor lombar crônica causa grande impacto na produtividade do trabalhador, afetando principalmente a qualidade de vida dos que sofrem dela. Ela pode gerar limitações funcionais complexas que tendem, em alguns casos, a acusar-se com o passar do tempo e produzir uma incapacidade permanente para atividades ocupacionais e ainda algumas atividades básicas cotidianas ${ }^{7}$. Sendo assim, verificou-se o nível de dor durante as atividades de casa e emprego demonstrando o impacto positivo da pesquisa na qualidade do trabalho dos indivíduos. $\mathrm{O}$ número de pessoas que conseguia realizar seus afazeres de casa/ emprego no começo da pesquisa sem dor alguma passou de $1(2,33 \%)$ para $12(27,91 \%)$ ao final da mesma.

Nesta pesquisa também foi realizada a coleta de dados referente às reações apresentadas pelos participantes ao longo dos seis encontros, após as aplicações. Dentre estas, as mais comuns foram, além da diminuição da dor lombar, melhora no sono em 16 casos $(37,31 \%)$, maior cansaço e sonolência durante 
o dia em 8 (18,60\%) casos, alterações no ciclo menstrual (tais como cólicas menstruais ou menstruação fora de época e diminuição dos sintomas da TPM) em 7 casos (16,28\%), alterações na coloração da urina em 15 casos (34,88\%), maior frequência da urina em 9 casos (20,93\%) e a resolução de outras dores que não apenas a lombar em 5 casos (11,63\%). Também foi relatado em 11 casos $(25,58 \%)$ que a dor retornou assim que os Stipers foram retirados dos pés (Tabela 4).

Observando os resultados individuais, dos 43 participantes, 7 (16,28\%) obtiveram melhora completa com relação a dor, tanto na escala facial de Wong Baker quanto no questionário Oswestry, somando 0 pontos em ambos os testes. Para os demais resultados, foi realizada a porcentagem de melhora, comparando ambos os valores do questionário Oswestry, obtendo-se: melhora de 99 a 75\% em 7 (16,28\%) indivíduos, de 74 a 50\% em 12 (27,91\%) casos, de 49 a $25 \%$ em $8(18,60 \%)$ casos, de 24 a $1 \%$ em 7 (16,28\%) casos e nenhuma alteração em $2(4,65 \%)$ pessoas (Tabela 5). Desta forma, a média geral de melhora do grupo foi de 55\% com relação a incapacidade gerada pela dor lombar.

Cabe ressaltar que, mesmo havendo dois participantes que não obtiveram resultados positivos no questionário Oswestry, permanecendo com o mesmo nível de incapacidade, ambos relataram diferença na intensidade da dor, mesmo que pouca, baseado nas escalas faciais de Wong-Baker, o que demonstra que todos os participantes tiveram resultados positivos com a intervenção.

\section{CONSIDERAÇÕES FINAIS}

A dor lombar pode atingir qualquer pessoa, independente de sexo ou idade e representa uma das mais frequentes disfunções em todo o mundo ${ }^{29,33}$. A lombalgia também causa incapacidade e sofri- mento $^{31}$, e precisa de cada vez mais estudos que proponham práticas efetivas para que esta seja sanada. Sendo assim, é pertinente a realização de pesquisas a respeito de técnicas não invasivas, simples, de baixo custo e com poucos efeitos colaterais, como as práticas naturais.

Através deste estudo, foi possível constatar que a utilização do Stiper em pontos reflexos da região lombar possui efeitos significativos na diminuição da dor e da incapacidade que esta causa, com a utilização de um produto simples, indolor e de efeito rápido. No entanto, seria interessante a realização de outra pesquisa, desta vez com um grupo controle, para que se possa comparar os resultados entre um grupo e outro, e assim verificar a verdadeira eficiência desta combinação de práticas.

Uma porcentagem baixa dos participantes fez uso de analgésicos durante a intervenção, portanto o resultado não foi significativamente comprometido. No entanto, para resultados mais fidedignos de futuras pesquisas, seria interessante admitir apenas pessoas que não façam uso constante destes medicamentos, mesmo porque foram justamente estes participantes que mostraram pouca ou nenhuma melhora no quadro de dor e incapacidade. Também foram estes que possuíam desvios da coluna, reumatismo e hérnias de disco. Ou seja, notou-se que a recuperação dos indivíduos que possuíam lesões na coluna foi menor comparada a quem relatou ter uma coluna saudável.

Em resumo, a pesquisa atingiu o objetivo proposto, avaliando os efeitos da aplicação do stiper através da reflexoterapia sobre a dor lombar e a incapacidade que esta causa. Desta forma, vê-se quão interessante a utilização do Stiper pode ser tanto para o naturólogo, quanto para outros profissionais da área da saúde que desejem estender e ampliar os resultados dos tratamentos executados. 


\section{CONFLITO DE INTERESSES}

À empresa STIPER Brasil por fornecer as pastilhas de silício para que a pesquisa fosse realizada.

\section{AUTORIA}

Todo o artigo foi feito pela acadêmica Cristina Avelino sob orientação da professora Graciela Medeiros.

\section{FONTE DE FINANCIAMENTO}

Declararam não haver

\section{REFERÊNCIAS}

1 - ALBUQUERQUE, A. V. Lombalgia crônica sem ciatalgia: correlação entre o quadro clínico e a radiologia. Rev Neurocienc, v.16, n. 3, p.184-188, 2008.

2 - AZEVEDO, J; SILVA, J; RIBEIRO, D. Relação entre lombalgia e sobrepeso em praticantes de atividade física. ConScientiae Saúde. v.7, n. 4, p. 471-475, 2008.

3 - BURIGO, F; LOPES, S. Lombalgia crônica mecânica: estudo comparativo entre acupuntura sistêmica e pastilhas de óxido de silício. Rev. Bras. Terap. e Saúde, Curitiba, v.1, n.1, p.27-36, jul./ dez. 2010.

4 - CAILLIET, R. Compreenda sua dor de coluna: um guia para prevenção, tratamento e alívio. São Paulo: Artmed, 2002.

5 - CECIN, H.A. Diagnóstico e Tratamento de Lombalgias e Lombociatalgias, 2001. Disponível em http://projetodiretrizes.org. br/projeto_diretrizes/072.pdf. Acesso em 18 de agosto de 2011.

6 - CENTRO COCHRANE DO BRASIL (CCDB). Nucleoplastia para o tratamento de hérnia discal. São Paulo, 2006.

7 - CHICAIZA, J.Aplicación de un programa de Reflexología podal para el manejo del dolor lumbar crónico de origen biomecánico. Colombia Umbral Científico ed: Departamento De Publicaciones Umb v.16 fasc.N/A p.17 - 29, 2011.

8 - CHOU, R; HUFFMAN, L. Nonpharmacologic Therapies for Acute and Chronic Low Back Pain: A Review of the Evidence for an American Pain Society/American College of Physicians Clinical Practice Guideline. Annals of Intern Medicine. vol.147, n.7, p.492504, 2007.

9 - CIDRAL FILHO, F. J. Naturologia Aplicada à qualidade de vida. In: HELLMANN, F; WEDEKIN, L.M. (Orgs.) 0 livro das interagências: estudo de caso em naturologia. Tubarão: Ed Unisul, 2008b.

10 - COSTA, D; PALMA, A. O efeito do treinamento contra resistência na síndrome da dor lombar. Rev. Port. Cien. Desp, vol.5, n.2, p.224234, mai, 2005.

11 - CRESWELL, J.W. Projeto de pesquisa: métodos qualitativo, quantitativo e misto. 2. ed. Porto Alegre: Artmed, 2007.

12 - FAIRBANK, J.; PYNSENT, P. The Oswestry disability index. Spine. v.25, n.22, p.2940-2953, 2000.

13 - FERNANDES, M. S., Lombociatalgia. Monografia (estágio supervisionado de Fisioterapia clínica), Faculdade Estácio de Sá, Campo Grande, novembro, 2007.

14 - GIL, A. C. Como elaborar projetos de pesquisa. 7. ed. São Paulo: Atlas, 2002.

15 - GILLANDERS, A. Guia completo de reflexologia: todo o conhecimento necessário para adquirir competência profissional. São Paulo (SP): Pensamento, 2008.

16 - HELLMANN, F; WEDEKIN, L.M. (Orgs.) 0 livro das interagências: estudo de caso em naturologia. Tubarão: Ed Unisul, 2008b.

17 - HELLMANN, F; WEDEKIN, L; DELLAGUSTINA, M,; MARANHÃO; A.L.; SILVA, F.M.; MARTINS, G.T.; GOULART, R (Org.). Naturologia Aplicada: Reflexões sobre saúde integral. Tubarão: Ed Unisul, 2008a.
18 - HOCKENBERRY, M.; WILSON, D. Wong's Essentials of Pediatric Nursing, ed 8. St. Louis: Mosby, 2007.

19 - JAYSON, M.; SMITH, T. Dor nas costas. São Paulo: Três, 2001

20 - KLAUMANN, P; WOUK, A; SILLAS, T. Patofisiologia da dor. Archives of veterinary science. V.13, n.1, p. 1-12, 2008.

21 - KWANG, W. T. Stiper: A fantástica acupuntura sem agulhas (Jornal o Legado de Alberto Sugamele - Abril/2006). Disponível em: www. stiper.com.br/site. Acesso em 20 set 2011].

22 - LAKATOS, E. M; MARCONI, M.A. Técnicas de pesquisa científica. 3. ed. São Paulo: Atlas, 2007.

23 - LIMA, V. Efeitos de um programa de exercícios físicos no local de trabalho sobre a flexibilidade e percepção de dor musculoesquelética entre trabalhadores de escritório. Faculdade de Medicina, Fisiopatologia Experimental. São Paulo, 2009.

24 - LOESER, J. D, TREEDE, R. D. The Kyoto protocol of IASP basic pain terminology. Pain. v.137: 473-477, 2008.

25 - LOURENÇO, O. T. Reflexologia podal: (primeiros socorros e técnicas de relaxamento). São Paulo: Ground, 2002.

26 - MACIOCIA, G. Os fundamentos da medicina chinesa: um texto abrangente para acupunturistas e fisioterapeutas. 2. ed. São Paulo: Roca, 2007.

27 - NEGRELLI, W.F., Hérnia discal: procedimentos de tratamento. Acta Ortop. Bras., v.9, n.4, p. 39-45, out./dez. 2001.

28 - PIRES, M; DUMAS, F.. Lombalgia: revisão de conceitos e métodos de tratamentos. Universitas: ciências da saúde [Online] v. 6, n. 2, p. 159-168, jul-dez, 2008.

29 - POLITO, M; NETO, G; LIRA, V. Componentes da aptidão física e sua influência sobre a prevalência de lombalgia. R Bras Ci e Mov, v. 11, n. 2, p. 35-40, 2003.

30 - PONTE, C. Lombalgia em cuidados de saúde primários: sua relação com características demográficas. Revista portuguesa de clínica geral, v. 21, p. 259-267, 2005.

31 - QUINTANILHA, A. Coluna vertebral: segredos e mistérios da dor. 2. ed. Porto Alegre: AGE, 2002.

32 - SAVEGNAGO, L. Estudo dos mecanismos envolvidos na ação antinociceptiva causada pelo disseleneto de difenila em camundongos. Tese de doutorado, Universidade Federal de Santa Maria, Santa Maria, 2007.

33 - SILVA, M.C.; FASSA, A.C.; VALLE, N.C. Dor lombar crônica em uma população adulta do Sul do Brasil: pravalência e fatores associados. Cadernos de saúde pública. Rio de Janeiro, v. 20, n.2, p. 233-385, mar./abr. 2004.

34 - TOSCANO, J., EGYPTO, E.P. A influência do sedentarismo na prevalência de lombalgia. Revista Braileira des Medicina do Esporte; vol.7, no. 4, p. 132-137, 2001.

35 - VITOR, A. O. et al. Psicofisiologia da dor: uma revisão bibliográfica - RECIIS - R. Eletr. de Com. Inf. Inov. Saúde. Rio de Janeiro, v. 2 $n^{\circ} 1: 87-96$, jan/jun. 2008 